\title{
Inhibitors of Tyrosine Kinases (TKI) and Small Interfering RNAs (siRNA) are Promising Targeted Cancer Treatments
}

\section{Haakon Skogseth ${ }^{1 *}$ Kåre E. Tvedt ${ }^{1}$ and Jostein Halgunset ${ }^{1,2}$}

${ }^{1}$ Department of Laboratory Medicine, Children's and Women's Health, Faculty of Medicine, Norwegian University of Science and Technology, Trondheim, Norway ${ }^{2}$ Department of Pathology and Molecular Genetics, St. Olav's Hospital, Trondheim, Norway

\begin{abstract}
Background: In recent years, many investigators have focused on the development of drugs with the potential to influence the malignant phenotype. Clearly, our understanding of molecular and cellular processes behind such mechanisms has increased substantially through the past decades. This knowledge has led to an extensive search for targeted carcinoma treatment.

Methods: This review is based on a qualified selection of articles published in internationally recognized journals. Some considerations are also based on the authors' own experience in clinical and molecular-targeted basic research.

Results: Inhibitors of tyrosine kinases (TKIs) and selective gene expressional regulators, small interfering RNAs, give hope for advances in the treatment of various carcinomas. However, it seems clear that the main challenge also in such therapy is the delivery, toxicity, uptake, and stability of small targeted molecules. These must be dealt with in order to unleash the potential of individual therapy.

Conclusion: Development of TKIs and siRNAs, with effects better than or at least similar to conventional therapy must be given high priority. Tolerable side effects are also critical to successful implementation. Only through such efforts may such treatment be fully developed and find its place in the treatment of malignant tumours, solely, as primary choice, or in combination with conventional cancer therapy.
\end{abstract}

Keywords: Carcinoma, TKI, Invasion, siRNA, Adhesion and Migration

\section{Introduction}

Cancer is primarily treated with chemotherapy, radiation, and surgery, alone or in combination, according to disease extent and prognosis. However, since the underlying mechanisms behind malignant cells are genetic, subsequent over-expressed or dysfunctional proteins, such therapy can not be considered as targeted cancer treatment. Moreover, up to now, advances in cancer treatment have been achieved by refining of conventional therapy. A few years ago almost all cancer drugs were directed against metabolic enzymes or the stability of the DNA strand. Late in the 1990s therapy targeting DNA topoisomerases had some success [1]. In addition to such therapy, the very common cancers of the breast and prostate, have been treated by interfering with hormonal signaling pathways via nuclear hormone receptors. Interestingly, it looks like a distinction in the cancer treatment field is about to happen these days, in respect to a better understanding of cancers' biology. Despite a wealth of different mediators that influence cancer development, cancer research seems to have found great hope in oncogenes that code for receptor proteins and their ligands. In particular, growth factors have been in the focus of intense research in recent years, and receptors of growth factors emerge as targets for effective therapy providing significant clinical benefits [2]. In addition to the obvious role of growth factors in cell growth and differentiation, examples of growth factors that regulate vascular formation seem to be targeted alternatives to conventional cancer treatment. In contrast, socalled junk DNA and epigenetic mechanisms such as DNA methylation and RNA-interference have received considerable attention, in the sense of being able to regulate gene expression, mainly posttranscriptional [3]. These highlights include the regulation of growth factor receptors and post transcriptional modification of gene products. For instance, microRNAs (miRNAs) have received significant attention as non- coding segments in regulation of gene expression, and some of them have been shown to take part in the development and progression of various cancers. In this review we summarize the role of TKI and miRNAs with a focus on their possible roles in cancer therapy.

\section{Tyrosine phosphorylation in cell behavior}

Protein tyrosine kinases (TKs) are enzymes that catalyze the transfer of phosphate groups from adenosine triphosphate (ATP) to tyrosine residues on specific protein substrates. Human cells contain more than 500 TKs [4], which play an important role in diverse cellular regulatory processes [5-8]. They work as mediators of signals leading to cell proliferation, differentiation, and migration, as well as to cell death [9]. There are two main classes of TKs, receptor bound and nonreceptor bound.

Growth factor receptors, which are transmembrane molecules composed of an extracellular ligand site, a transmembrane adaptor and an intracellular domain with enzymatic activity, are activated by binding an extracellular signal molecule, such as a growth factor (Figure 1). For instance, the EGFR family consists of four related

*Corresponding author: Haakon Skogseth, Department of Laboratory Medicine, Children's and Women's Health, Faculty of Medicine, Norwegian University of Science and Technology, St. Olav's Hospital7006 Trondheim, Norway, Tel: +47 72573081, E-mail: haakon.skogseth@ntnu.no

Received August 17, 2011; Accepted November 23, 2011; Published Novembe 26, 2011

Citation: Skogseth H, Tvedt KE, Halgunset J (2011) Inhibitors of Tyrosine Kinases (TKI) and Small Interfering RNAs (siRNA) are Promising Targeted Cancer Treatments. J Carcinogene Mutagene 2:122. doi:10.4172/2157-2518.1000122

Copyright: (C) 2011 Skogseth $\mathrm{H}$, et al. This is an open-access article distributed under the terms of the Creative Commons Attribution License, which permits unrestricted use, distribution, and reproduction in any medium, provided the original author and source are credited. 


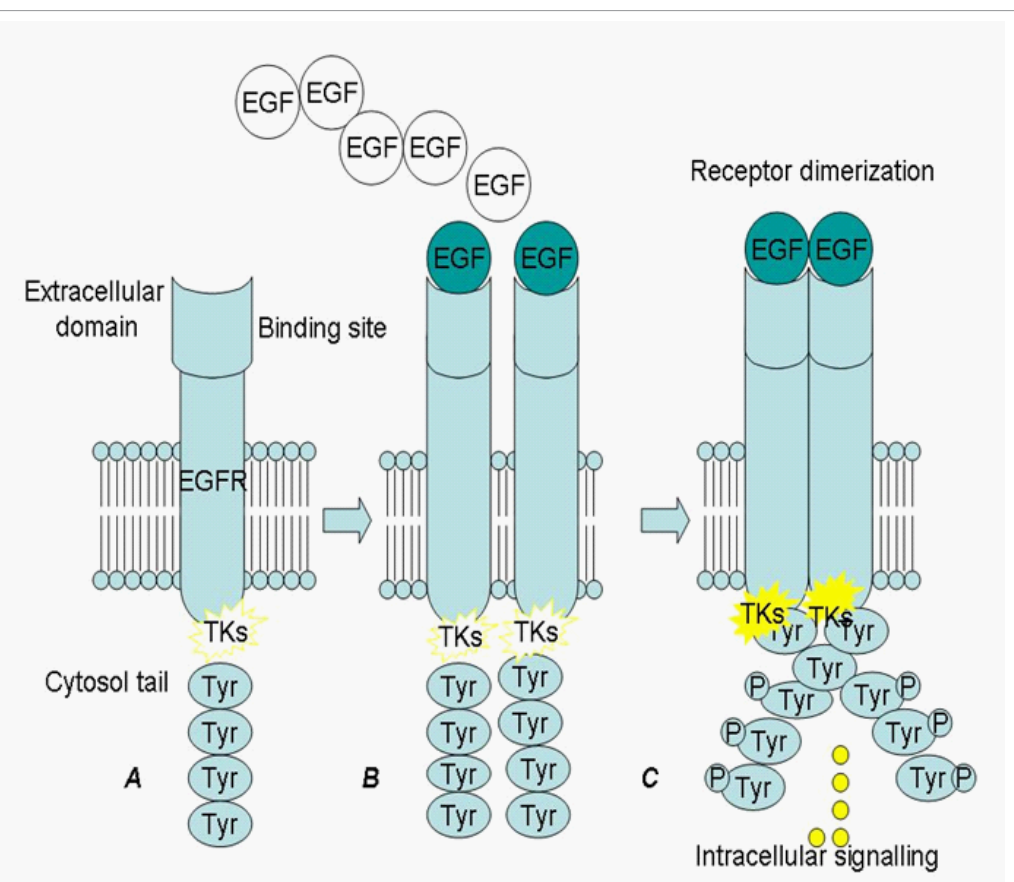

Figure 1: Structure (A) and activation (B) of a typical tyrosine kinase receptor, the receptor binding epidermal growth factor (EGF). These receptors have one transmembrane segment. The extracellular portion of the receptor binds the ligand (EGF in this case). Inside the cell, a portion of the receptor has tyrosine kinase activity. The remainder of the receptor contains a series of tyrosine residues that are substrates for the tyrosine kinase. The activation of receptor tyrosine kinases starts with the binding of a messenger, causing receptor aggregation or clustering. Once the receptors aggregate, they cross-phosphorylate each other at a number of tyrosine amino acid residues (C). The formation of tyrosine phosphate (Tyr-P) residues on the receptor creates binding sites for cytosolic SH2 domains.

transmembrane receptors that are involved in the regulation of cellular growth and differentiation. In the absence of a ligand, a receptor TK is unphosphorylated and monomeric. When a ligand binds to the extracellular domain of the receptor, an oligomerization of receptors takes place, which in turn leads to the phosphorylation of regulatory tyrosines [10-12]. Multiple cytoplasmic signalling pathways, including the rat sarcomas (RAS) mitogen-activated protein kinase pathways, the phosphoinositol 3-kinase (PI3K) pathway, and the protein kinase C pathway, may then be activated. TK signaling is terminated through the action of tyrosine phosphatases or by other inhibitory intracellular molecules.

The non-receptor TKs are cytoplasmic proteins, exhibiting considerable structural variability. They are for instance known as mediators in Src homology-2 (SH2) and Src homology-3 (SH3) signaling pathways, and are maintained inactive by intracellular inhibitor proteins. They are activated when these inhibitors are dissociated from the enzyme [13].

\section{Dysfunctions of TKs}

Enhanced expression of EGFR or its ligands such as EGF and transforming growth factor (TGF- $\alpha$ ) can increase signaling via receptor-mediated pathways that may lead to excessive proliferation and cellular transformation. Several studies have shown that EGF binding to EGFR on tumor cells blocks apoptosis, and consequently promotes tumor growth and viability. Moreover, EGFR and its ligands have an important role in regulating angiogenesis [14-17]. Numerous studies have demonstrated that TKs are dysregulated in cancer cells, in various ways. Dysfunctions in growth factor signal cascades probably represent a main characteristic in the progression of malignant cell behavior [18-21].
Normally, the level of intracellular protein phosphorylation is tightly controlled. However, in cancers, various TK dysregulations occur (Table 1), such as uncontrolled expression of TK-receptors or their ligands [22]. An abnormality frequently seen is the fusion of TKreceptors with partner proteins, resulting in constitutive oligomerization in the absence of ligands. In this way autophosphorylation is promoted, resulting in uncontrolled activation of growth responses [23-26]. Another important mechanism in irregular activation of TKs involves mutations that disrupt the autoregulation of the kinase [27]. Finally, increased TK activity may be due to a decrease of factors that limit TK activity, such as tyrosine phosphatases or other TK inhibitory proteins [28]. The network of regulatory pathways involving TKs is exceedingly complex, and its ramifications are not yet known in great detail. However, it seems beyond doubt that fundamental cellular processes, such as growth, survival, differentiation and motility, are largely determined by the phosphorylation status of key control proteins, and that these signal systems may be extensively interwoven.

\section{TKI therapy in clinical trials}

The two classes of growth factor receptors of clinical significance are the EGF-family and VEGFR. The first class is further divided into EGFR, ERBB1-4. Except for ERBB3, all these receptors are based on tyrosine kinase phosphorylation. Moreover, they are shown to be oncogenes. Quite differentlt, in the recruitment of new blood vessels induced by the three subclasses of VEGF-familiy, namely the VEGFR 1-3, it is the ligand binding to the specific receptor that play the main role. However, since cancer is commonly understood as the result of dysregulation of cell growth, anti-cancer drugs have mainly been sought among chemical substances directly inhibiting the cellcycle or interfering with signal systems promoting cell proliferation. 
Citation: Skogseth H, Tvedt KE, Halgunset J (2011) Inhibitors of Tyrosine Kinases (TKI) and Small Interfering RNAs (siRNA) are Promising Targeted Cancer Treatments. J Carcinogene Mutagene 2:122. doi:10.4172/2157-2518.1000122

\begin{tabular}{|c|c|c|}
\hline Tyrosine kinase & Mechanism & Examples \\
\hline \multirow[t]{3}{*}{ EGFR (ErbB1) } & Mutation (EGFR-vIII) & Gliomas \\
\hline & Mutations in TK-domain & Non-small cell lung cancer \\
\hline & $\begin{array}{l}\text { Over-expression or growth factor mediated } \\
\text { activation }\end{array}$ & $\begin{array}{l}\text { Head- and neck, lung-, breast-, prostate- and colorectal } \\
\text { cancer }\end{array}$ \\
\hline HER2 (ErbB2) & Over-expression (e.g. amplification of the gene) & Breast-, ovary- and lung cancer \\
\hline $\begin{array}{l}\text { c-Kit (cellular homolog of the feline sarcoma viral } \\
\text { oncogene) }\end{array}$ & Mutation (constitutive TK activity) & Gastrointestinal stromal tumors (GIST) \\
\hline PDGFR (platelet-derived growth factor receptor) & Mutations & Fibrosarcoma, chronic myelomocytic leukaemia \\
\hline \multicolumn{3}{|l|}{ Non-receptor tyrosine kinases } \\
\hline Bcr-Abl (Breakpoint cluster region-Abelson) & Mutation (translocation) & Chronic myeloid leukemia \\
\hline
\end{tabular}

Table1: Examples of dysregulated tyrosine kinases in various cancer types [29-31].

The development of growth factor receptor inhibitors has recently become a central field of research. However, despite the development of several chemicals with such properties, mainly tyrosine kinase inhibitors (TKIs), the presently available drugs serve as second or third line therapy rather than constituting a primary choice. Various TKIs have been used in clinical trials, and their effects have been thoroughly reviewed. In summary, treatment with TKIs has shown positive effects in patients with non-small cell lung cancer, breast cancer, colorectal cancers, and in patients with leukemia, especially when given in combination with conventional cytostatics or radiation therapy. Not surprisingly, the best results are obtained when the TKs are mutated and permanently active, for instance in chronic myeloid leukaemia. There are also promising results with TKIs in the treatment of HER-2-positive breast cancer. Moreover, TKIs have been shown to counteract other aspects of malignant disease, such as neoangiogenesis [32-34]. The benefit of targeted therapy with herceptin, avastin and tykerb is well proven in breast cancers, while gleevec has been used in the treatment of GISTs and chronic myeloid leukemia. Tarceva and iressa have been used with NSCLC, and sutent and nexavar show promising effect in targeted therapy to renal cell carcinoma. Moreover, vectibix and erbitux have been second line therapy in the treatment of colorectal cancers. A very interesting observation in TKI treatment is the extreme variations in inter-individual response to many TKIs, however they seem to be well tolerated. Identified factors such as age, diet, smoking, the intake of alcohol, gender and renal/liver function together with genetic polymorphisms, have been shown to modify their pharmacokinetics. It is a main challenge to individualize the treatment of cancer with the eventually multifaceted group of TKIs and antibodies to growth factor receptors.

\section{Treatment with small interfering RNAs (siRNA)}

In clinical trials, the drugs still seem to be well-tolerated with fewer side effects than conventional cancer therapy. The most frequent side effects reported are mild skin rash and brief diarrhoea. However, the high rate of mutations in many cancer cells creates an additional problem which must be considered, namely the ability of cancer cells to acquire drug resistance. As TKIs gradually have been introduced in cancer therapy, this has become a great challenge, and researchers have therefore sought for highly specific but non-toxic drugs in an attempt to inhibit the development and progression of carcinomas.
Recent research takes advantage of the mechanisms underlying the so-called RNA interference. Andrew Fire and Craig Mello were awarded the 2006 Nobel Prize, for their description of how small RNA fragments could prevent translation of mRNA, thereby suppressing the expression of certain genes [35]. Introduction of double stranded RNA homologous to a specific gene in the round worm Caenorhabditis elegans, resulted in post- transcriptional suppression of various genes, and the phenomenon has since been found in other organisms such as humans. It quickly aroused great expectations as to its therapeutic potential, and the biological mechanisms of such RNA fragments were thoroughly investigated.

RNAi is a response triggered by the introduction of synthetic double stranded RNA molecules (dsRNA) into eukaryotic cells. As a response to the dsRNA, the cells inhibit the expression of genes containing the corresponding base sequence. The process starts with the cleavage of dsRNA into short segments, 21-23 base pairs (bp) long, each with 2 -necleotide overhangs on the 3 ' end. This cleavage is catalyzed by the adenosine triphosphate (ATP) dependent enzyme complex designated Dicer, localized in the cytoplasm. Next, the double stranded siRNAs bind to a nuclease complex and form what is called an RNA-induced silencing complex (RISC). Unwinding of the siRNAs is necessary for the activation of RISC, which then targets the homologous mRNA by base pairing interactions. At least, RISC cleaves the mRNA, resulting in silencing of the gene that code for the mRNA in question [36]. RNAi seems to have arisen as a protective mechanism for cells against dsRNA, for instance from dsRNA viruses [37]. When utilizing the RNAi response to down-regulate the expression of a protein, a complication is seen with the administration of long dsRNAs to mammalian cells, where a so-called interferon response is trigged. This response has probably evolved as an additional defense mechanism against dsRNA containing viruses, and results in the complete shutdown of protein synthesis in the cell. To avoid this response, it is therefore crucial to administer dsRNAs that are not longer than 30 nucleotides. When administering foreign dsRNA to mammalian cells, the dsRNA used must therefore be siRNA [36]. Moreover, RNAi -mediated reduction of protein expression is usually obtained by transfection with synthetic oligonucleotides. Transfection denotes processes whereby foreign DNA or RNA is administered to cells [36]. Thermodynamic properties determine which RNA strand is loaded into RISC. This and other sequence criteria underlie different algorithms for selection of 
a synthetic siRNA to reduce the expression of a certain protein [38]. Manufacturers of synthetic siRNAs have computer programs based on such algorithms to return different siRNA sequences, each given a score as to their expected efficiency in reducing the expression of the target protein. In addition, companies designing siRNAs often provide each siRNA with control siRNA. Such siRNA are similar in sequence to their corresponding siRNAs, but with some mismatches. They are designed to minimize the sequence homology to any known vertebrate transcript, and are not supposed to induce interferon-mediated stress response pathways. The control siRNAs are therefore not supposed to be effective in down regulating the expression of any particular protein, and are used as a control to see whether or not the depression is specific for the desired protein or a result of stress caused by the transfection procedure itself.

In addition to being a useful tool to study various gene functions, it is also hoped that RNA interference can be used therapeutically in the future. Malignant cells show an increased expression of many proteins necessary for invasion and metastasis. These include proteins with effects on adhesion, proteolysis, proliferation, and formation of new blood vessels. One possibility for the therapeutic use of RNA interference will be to stop the production of one or more proteins that increase the cell's malignant potential, thus preventing further growth and spread of tumors. Animal experiments with intratumoral injections of siRNA have also shown that tumor size and the tendency to metastasis is reduced by knocking out the production of proteins involved in tumor growth and proliferation. RNAi can be induced in cells by adding small synthetic dsRNA, or viral vectors that express small stem-loop RNA, called "short hairpin" RNA (shRNA). Intracellular dsRNA is processed to siRNA while the shRNA is processed as miRNA [39]. The process by which RNA molecules are added to cells is called transfection, and cells that have inserted a foreign siRNA are designated tranfected cells.

RNAi research has evolved to become an important tool for studying the function of many cellular proteins and how the loss of these proteins will affect the organism. The technique is alternative to creation of transgenic organisms and cell lines of knock-out phenotypes. However, among other things, it was early discovered that introduction of long double RNA sequences initiated an antiviral interferon response in mammalian cells. To avoid the kind of response, very short single-RNA sequences were applied in studies of mammalian gene functions. In addition to antiviral reactions, the transport of siRNA to the target cells remains a main challenge. Toxic effects and inefficient uptake of the RNA sequences are also methodological difficulties, and the latter has lead to a continuous development of new vectors for the introduction of synthetic RNA [40,41] and [42-46]. Briefly summarized it has been shown that miRNAs can function as tumor suppressors as well as tumor promoters, and that the aberrant expression of miRNAs is associated with the stage, progression and metastasis of various cancers. However, the realization of miRNA-based therapy in the clinic may be far ahead, since a lot of problems related to delivery, stability, specificity of the small oligonucleotides must be further elucidated and overcome.

\section{General Discussion}

Cancer is commonly understood as the result of deregulation of cell growth, and anti-cancer drugs have mainly been sought among chemical substances directly inhibiting the cell-cycle, or interfering with signal systems promoting cell proliferation. Despite extensive research into the biology of tumours, which has led to ever increasing knowledge about the underlying mechanisms [47-50], a major breakthrough in the treatment of this disease category has yet to appear. Overall, advances in clinical treatment of patients have up to now been achieved by optimization of available conventional therapies, most of which have been used for many years. In the future, clinical handling of cancer will increasingly rely on the division of the patient group into subgroups of different risk for progressive disease and formation of metastases. Based on such information patients will be selected for individualized therapy, aimed at preventing the spread of cancer cells, giving to each patient the treatment necessary to control the disease, nothing more, nothing less. The selection of a suitable therapy will mainly be based on extensive evaluation of biopsies. This field has been reviewed several times, for example by Timmons and collaborators [35]. Moreover, for future development of new therapies, it should be kept in mind that the biological machinery is fundamentally the same in normal and transformed cells. This means that conventional anti-cancer drugs, which are essentially anti-proliferation drugs, will inevitably produce deleterious effects also in actively proliferating normal cell populations. Thus, the risk of unwanted side effects is usually the factor limiting the extent of the treatment.

TKIs have been used with impressive success in some selected tumour types with tyrosine kinase hyper function as a characteristic and essential element in the pathology, although toxic side-effects continue to be a problem. It turns out that many of the processes which are characteristic of the malignant phenotype may be modified by inhibition of various TKs, and that meticulously selected tyrosine kinase inhibitors therefore may have a role to play as anti cancer drugs. A number of investigators have suggested that TKIs have the potential to modulate the invasive capacity of carcinoma cells, especially in tumours with dysregulated growth factor receptor pathways [51,52]. TKIs which have been carefully designed and selected to suppress various aspects of the cells' invasive behaviour, might be expected to show less general toxicity than ordinary cytotoxic drugs, because of their relative specificity for malignant cells. In keeping with this idea, in vitro studies have demonstrated that the invasive property of prostatic cancer cells can be modulated by some TKIs. In such studies, the invasive capacity of aggressive variants of prostate cancer cells is more heavily modified by TKIs than that of more indolent types. Moreover, TKs appear to be especially effective inhibitors of overexpressed proteins, for example uPA in PC-3 cells. However, presently available TKIs are not the result of a systematic search in this direction, and therefore the major challenge is still to discover compounds with a higher effect: toxicity-ratio. Recent research gives reason to hope that it may be possible to find TKIs with specific inhibitory effect on cancer cell invasion. Thus, in an in vitro invasion model using prostate cancer cells, measurement of caspase activation revealed no sign of serious cell injury at TKI doses which produced a dramatic reduction of the cells invasive capacity [27]. This supports the notion that the observed effect is a specific interference with the invasive behaviour and not merely the result of general cytotoxicity, thus extending the well known effects of inhibition of cell proliferation and apoptosis induction caused by TKI in cultured cells [53]. However, the usefulness of TKIs in the treatment of patients will probably depend on improving their pharmacokinetics in terms of plasma stability and, preferably, a more precise uptake by target cells. Strategies for achieving efficient systemic delivery of the drugs may therefore be important for their eventual success.

Also small interfering RNAs constitute a class of promising candidate drugs for cancer treatment. Because of its relative short history, it is too early to predict the therapeutic usefulness of RNA interference, although there is evidence that it may produce selective 
Citation: Skogseth H, Tvedt KE, Halgunset J (2011) Inhibitors of Tyrosine Kinases (TKI) and Small Interfering RNAs (siRNA) are Promising Targeted Cancer Treatments. J Carcinogene Mutagene 2:122. doi:10.4172/2157-2518.1000122

post-transcriptional inhibition in vivo. Thus, down-regulation of different proteins has been shown to reduce the tumor growth rate, and has in some cases also led to a reduced tumor size [1,2]. In any event, the efficient delivery of such drugs to the target cells will constitute a challenge of vast proportions. Targeted delivery supposes the effective, precise and safe distribution of the drug, with a minimum of systemic adverse effects. Most existing transfection reagents have too many and too serious side effects to be real alternatives in clinical oncology. If the method can be rendered sufficiently effective and of acceptable toxicity, it could be a breakthrough in the treatment of cancers that currently can not be treated effectively, such as carcinomas which are resistant to conventional treatment. All taken into consideration, RNA interference is likely to become a treatment option for some cancers, either alone or as a supplement to traditional treatment. For RNA interference to become an established treatment for cancer, it will be necessary to have multiple possible targets, since tumors are very heterogeneous, but also considering the wide variation among cells within a single tumor. However, similar dilemmas are generally raised in most systemic treatment strategies.

\section{References}

1. Salerno S, Da Settimo F, Taliani S, Simorini F, La Motta C , et al. (2010) Recent advances in the development of dual topoisomerase I and II inhibitors as anticancer drugs. Curr Med Chem 17 4270-4290.

2. Larsen AK, Ouaret D, El Ouadrani K, Petitprez A (2011) Targeting EGFR and $\operatorname{VEGF}(R)$ pathway cross-talk in tumor survival and angiogenesis. Pharmacol Ther 131: 80-90.

3. Melnyk CW, Molnar A, Baulcombe DC (2011) Intercellular and systemic movement of RNA silencing signals. EMBO J 30: 3553-3563.

4. Hubbard SR (1999) Structural analysis of receptor tyrosine kinases. Prog Biophys Mol Biol 71: 343-358.

5. Heldin CH (1996) Protein tyrosine kinase receptors. Cancer Surv 27: 7-24.

6. Pawson T (2002) Regulation and targets of receptor tyrosine kinases. Eur J Cancer 38: S3-10.

7. Schlessinger J (2000) Cell Signaling by receptor tyrosine kinases. Cell 103: 211-225.

8. Bazley LA and Gullick WJ (2005) The epidermal growth factor receptor family. Endocr Relat Cancer 12: S17-27.

9. C. H. Heldin (1995) Dimerization of cell surface receptors in signal transduction. Cell 80: 213-223.

10. Michell RH (1987) How do receptors at the cell surface send signals to the cell interior? Br Med J (Clin Res Ed) 295: 1320-1323.

11. Jiang G, Hunter $T$ (1999) Receptor signalling: when dimerization is not enough. Curr Biol 9: 568-571.

12. Tsygankov AY (2003) Non-receptor protein kinases. Frontiers in Bioscience 8: s595-635.

13. Woodburn JR (1999) The epidermal growth factor and its inhibition in cancer therapy. Pharmacol Ther 82: 241-250.

14. Perry JE, Grossmann ME, Tindall DJ (1998) Epidermal growth factor induces cyclin D1 in human prostate cancer cell line. Prostate 35: 117-124 .

15. Wells A (1999) EGF receptor. Int J Biochem Cell Biol 31: 637-643.

16. van Cruijsen H, Giaccone G, Hoekman K (2005) Epidermal growth factor receptor and angiogenesis: Opportunities for combination anticancer strategies. Int J Cancer 117: 883-888.

17. Kolibaba KS, Druker BJ (1997) Protein tyrosine kinases and cancer. Biochim Biophys Acta 1333: 217-248.

18. Spencer-Cisek PA (2002) The role of growth factors in malignancy: a focus on the epidermal growth factor receptor. Semin Oncol Nurs 18: 13-19.
19. Ware JL (1998) Growth factor network disruption in prostate cancer progression Cancer Metastasis Rev 17: 443-447.

20. Tang CK, Lippman ME (1998) EGF family receptors and their ligands in human cancer, in Hormones and Signaling. (In O'Malley BW, ed.), 1Academic Press, San Diego, CA., pp. 113-165

21. Voldborg BR, Damstrup L, Spang-Thomsen M ,Poulsen HS(1997) Epiderma growth factor receptor (EGFR) and EGFR mutations, function and possible role in clinical trials. Ann Oncol 8: 1197-1206.

22. Nakao M, Yokota S, Iwai T, Kaneko H, Horiike S, et al. (1996) Internal tandem duplication of the flt3 gene found in acute myeloid leukemia. Leukemia 10: 1911-1918.

23. Lynch TJ, Daphne WB, Sordella R, Gurubhagavatula S (2004) Activating mutations in the epidermal growth factor receptor underlying responsiveness of non-small-cell lung cancer to gefitinib. N Engl J Med 350: 2129-2139

24. Pao W, Miller V, Zakowski M,Doherty J, et al. (2004) EGF receptor gene mutations are common in lung cancers from "never smokers" and are associated with sensitivity of tumors to gefitinib and erlotinib. Proc Natl Acad Sci USA 101: 13306-13311

25. Sordella R, Bell DW, Haber DA, Settleman J (2004) Gefitinib-sensitizing EGFR mutations in lung cancer activate anti-apoptotic pathway. Science 305: 11631167

26. Smith KM, Yacobi R, Van Etten RA (2003) Autoinhibition of Bcr-Abl through its SH3 domain. Mol Cell 12: 27-37

27. Haakon Skogseth, Erik Larsson, Jostein Halgunset (2005) Inhibitors of tyrosine kinase inhibit the production of urokinase plasminogen activator in human prostatic cancer cells. APMIS 113: 332-339

28. Mendelsohn J, Baselga J(2003) Status of epidermal growth factor receptor antagonists in the biology and treatment of cancer. J Clin Oncol 21: 2787-2799

29. Elbashir SM, Harborth J, Weber K, Tuschl T (2002) Analysis of gene function in somatic mammalian cells using small interfering RNAs. Methods 26: 199-213

30. Becker WM, Kleinsmith LJ, Hardin J (2003) The world of the cell. 5. ed, Benjamin Cummings, San Francisco, 30pp

31. Reece RJ (2004) Analysis of genes and genomes 1. ed, John Wiley \& Sons Ltd., West Sussex, 457 pp

32. Arora A, Scholar EM (2005) Role of tyrosine kinase inhibitors in cancer therapy J Pharmacol Exp Ther 315: 971-979

33. Herbst RS, Kies MS(2003) Gefitinib: current and future status in cancer therapy. Clin Adv Hematol Oncol 1: 466-472

34. Tang PA, Tsao MS, Moore MJ (2006) A review of erlotinib and its clinical use. Expert Opin Pharmacother 7: 177-193

35. Timmons L, Tabara H, Mello CC, Fire AZ (2003) Inducible systemic RNA silencing in Caenorhabditis elegans. Mol Biol Cell 7: 2972-2983

36. Shankar $P$, Manjunath N, Lieberman J (2005) The prospect of silencing disease using RNA interference. JAMA 293: 1367-1373

37. Aagaard L, Rossi JJ (2007) RNAi therapeutics: principles, prospects and challenges. Adv Drug Deliv Rev. 59: 75-86

38. Castanotto D, Sakurai K, Lingeman R, Li H, Shively L, et al. (2007) Combinatorial delivery of small interfering RNAs reduces RNAi efficacy by selective incorporation into RISC. Nucleic Acids Res 35: 5154-5164

39. Danen $\mathrm{EH}(2005)$ Integrins: regulators of tissue function and cancer progression. Curr Pharm Des 11: 881-891

40. Jana S, Chakraborty C, Nandi S, Deb JK (2004) RNA interference: potentia therapeutic targets. Appl Microbiol Biotechnol 65: 649-657

41. Shrivastava N, Srivastava A (2008) RNA interference: an emerging generation of biologicals. Biotechnol J 3: 339-353

42. Fornaro M, Manes T, Languino LR (2001) Integrins and prostate cancer metastasis. Cancer Metastasis Rev 20: 321-331 
Citation: Skogseth H, Tvedt KE, Halgunset J (2011) Inhibitors of Tyrosine Kinases (TKI) and Small Interfering RNAs (siRNA) are Promising Targeted Cancer Treatments. J Carcinogene Mutagene 2:122. doi:10.4172/2157-2518.1000122

Page 6 of 6

43. Hynes RO (1992) Integrins: versatility, modulation, and signalling in cell adhesion. Cell 69: 11-25

44. Yebra M, Goretzki L, Pfeifer M, Mueller BM (1999) Urokinase-Type plasminogen activator binding to its receptor stimulates tumor cell migration by enhancing integrin-mediated signal transduction. Exp Cell Res 250: 231-240

45. Huges C, Murphy A, Martin C, Sheils O and O'Leary J(2005) Molecular pathology of prostate cancer. J Clin Pathol 58: 673-684

46. Ashihara E, Kawata E, Maekawa T (2010) Future prospect of RNA interference for cancer therapies. Curr Drug Targets 11: $345-360$

47. Huang C, Li M, Chen C, Yao Q (2008) Small interfering RNA therapy in cancer: mechanism, potential targets, and clinical applications. Expert Opin Ther Targets 12: 637-645

48. Saksela O (1985) Plasminogen activation and regulation of pericellular proteolysis. Biochim Biophys Acta 823: 35-65

49. Veronese ML, Algazy K, Bearn L, Eaby B, Alavi J, et al. (2005) Gefitinib in patiens with advanced non-small cell lung cancer (NSCLC): the expanded access protocol experience at the University of Pennsylvania. Cancer Invest 23: 296-302

50. Gorre ME, Mohammed M, Ellwood K, Hsu N, Paquette R, et al. (2001) Clinica resistance to STI- 571 cancer therapy caused by BCR-ABL gene mutation or amplification. Science 293: 876-880

51. Arteaga C, Johnson DH (2001) Tyrosine kinase inhibitors-ZD1839 (Iressa) Curr Opin Oncol 13: 491-498

52. Li J, Kleef J, Giese N, Buchler MW, Korc M, et al. (2004) Gefitinb ("Iressa", ZD1839), a selective epidermal growth factor receptor tyrosine kinase inhibitor inhibits pancreatic cancer cell growth, invasion, and colony formation. Int $J$ Oncol 25: 203-210.

53. de Thonel A and Eriksson JE (2005) Regulation of death receptors-relevance in cancer therapies. Toxicol Appl Pharmacol 207: 123-132. 